palliative care servcies. Final results are due to complete by June 2013.

The project was part funded by the Burdett Trust for Nursing.

\section{P158 A HOSPICE MOTOR NEURONE DISEASE CLINIC: CHALLENGES AND BENEFITS OF A NEW SERVICE}

1,2Jane Bywater, ${ }^{1}$ Jo Butt, ${ }^{1}$ Caroline Mathias, ${ }^{1}$ Jean Smith, ${ }^{2}$ Rachel Gaffney. ${ }^{1}$ St Michaels Hospice, Basingstoke, United Kingdom, ${ }^{2}$ Hampshire Hospitals Foundation Trust

10.1136/bmjspcare-2013-000591.180

Background/context The Hospice in North Hampshire had offered support and care to MND patients but in an unstructured way. Close working between the Palliative Medicine and Neurology Consultants led to a recognition that both early and advanced care could be improved if services were to work more closely together.

Aim The aim was to set up and run a montly multi-disciplinary clinic for people with MND in the Hospice, allowing access to all members of the multi-disciplinary team (neurologist, consultant in palliative medicine, care centre co-ordinator, hospice at home team, social worker, physiotherapist, OT, speech and language therapist, Association Visitors) via one clinic. The clinic link with the MNDA would improve access to information about services. The outpatient hospice setting allows informal support for pts and carers from Assoc Visitors.

Outcomes To improve the patient and carer experience.

To ensure access to hospice services early in diagnosis.

To improve both peer and carer support through joint working with the MNDA.

To develop a specialist skill base with opportunities for support and training of other professionals.

To develop patient professional relationships which allow easy transition in the end stages of illness.

Application to hospice practice Many centres across the country run a multidisciplinary MND clinic but traditionally in the hospital settling. Evidence is good for improvement in patient care from a hospital model of service delivery model. Hospices have many advantages with their experience in coordinating care and community focus. The early links with the Hospice allow continuity of care throughout the patient journey.

\section{P159 CHANGING A LYMPHOEDEMA SERVICE THROUGH SERVICE REDESIGN}

Debbie Murphy. St Catherine's Hospice, Preston, UK

\subsection{6/bmjspcare-2013-000591.181}

Background Well established Lymphoedema service facing number of escalating issues:

- Increasing referrals

- Over budget

- Frustrated staff

- Lack of care pathways and long term management

- Lack of innovation

Aim

- Reduce financial strain

- Provide clear care management strategy

- Introduce self-management strategies

- Ensure full access for individuals with Lymphoedema.
Approach Used

- Poor team work - looked at strengths and the weaknesses of each team member as a team

- Cost reduction - compression garment formulary assessed on cost effectiveness, size of range, availability on FP10, education supplied by the company.

- Care pathways - patient journey plotted dependent on severity of condition.

- Discharge policy developed, packs sent to the GP and to patient.

- Long term self-management - six week education programme combined with fun exercise class (Le Bed), gives patients tools to manage own condition.

- Following discharge from Le Bed, Lymphoedema Information Fun Event (LIFE) established after feedback from patients - provides ongoing information about service development. Group used as patient reference and involvement group e.g. feedback on leaflets etc.

- Investment in new technologies (lymph assist machines, lasers, oscillator) - enhances treatments offered, produces quicker results.

- Kinesio tape introduced

- Children's Lymphoedema Service initiated

- Bra fitting service developed.

Outcomes

- Greater sense of team - clear lines of reporting, goal setting and monitoring.

- Expansion of team with a clear mix of skills

○ support worker undertaking more routine work, specialist nurses concentrate on more complex cases

$\circ$ part time specialist appointed

- Lymphoedema physiotherapist (still to be appointed)

$\circ$ training and development - manual lymphatic drainage, Le Bed instructor training, bra fitting, wound management, child protection training etc.

- Number of new patient referrals has increased year on year (see table)

- Negotiated and secured contracts with three additional CCG's

- $2008 / 2009=£ 23 \mathrm{k}$ overspend, 2011/2012 break even (including reinvestment in new technologies and education)

- Number of intensive treatments increased from 27 in 2010/2011 to 119 in 2012/2013

- Patients self-managing - the discharge pathway and Le Bed class - leading to greater self esteem

- Lymphoedema manager recognised as Lymphodema nurse of the Year 2013, British Journal of Nursing

P160 THINK AGAIN! TREATMENT CHANGES FOR PATIENTS WITH HEAD, NECK AND FACIAL SWELLING

Julie Norris. St Catherine's Hospice, Preston, UK

10.1136/bmjspcare-2013-000591.182

Background Few patients referred for head, neck and facial lymphoedema as widely viewed that very little could be done, apart from prescribing tight fitting compression garments which are very constrictive and have a low patient compliance.

Aim

- Improve access for patients experiencing lymphoedema to the head, neck and face.

- Identify alternative treatment methods 
- Develop closer working relationships with the Speech and Language, Dietician and Head and Neck services.

Approach Used Four cornerstones of care used:-

- Skin care: application of regular skin cream to hydrate skin, prevent infection and encourage self-management

- Manual Lymphatic Drainage (MLD): specialised gentle massage to aid and stimulate drainage.

- Simple Lymphatic Drainage; patient learns to move the build-up of fluid

- Exercises (facial):- Encourages muscle movement, aids movement of lymphatic fluid. Breathing exercises stimulate deeper lymphatic vessels in chest and abdomen to enhance lymph drainage.

- Compression worn to maintain shape, reduce swelling.

New treatment modalities (accompanied by modified MLD):

- Low level laser therapy: infra-red wavelength penetrates deep into tissues, aids softening of fibrosis and scar tissue, useful in treating cording.

- Deep oscillation therapy: - penetrates deep into body tissue triggering lasting resonance

- Kinesio Tape: increases body's ability to drain lymphatic drainage to healthy lymph nodes

These innovative treatments have proved to be an effective adjunct to our existing specialist knowledge and enhanced treatments offered to patients

Measurements pre and post treatment compared using photographs

Patients complete questionnaire after each session, record any immediate changes to symptoms.

Outcomes Increased referrals for treatment to head, neck and face

$2011 / 2012$ - 5 referrals

2012/2013 - 17 referrals

Development of a closer working relationship with the surgical head and neck multidisciplinary team at hospital.

Patient comments I don't feel that everyone is looking at my face as they did before, I felt like I was the elephant man, much happier and I went for a walk with the wife, I wouldn't have done that a few weeks ago.

I was able to eat normal food after treatment for that day

\section{P161 MEANING OF PAIN FOR PATIENTS WITH ADVANCED CANCER AND HOW IT INFLUENCES BEHAVIOUR: A QUALITATIVE RESEARCH STUDY}

Rebecca Jennings. St Joseph's Hospice, London, United Kingdom

10.1136/bmjspcare-2013-000591.183

Background Health related quality of life incorporates physical function and independence which are high priorities for patients with advanced cancer. These two inter-related domains can be compromised through the experience of cancer-related pain. Patient-held meanings of cancer pain may have potential to influence behaviours which determine functionality and quality of life.

Study aim To explore the meanings of pain among patients living with advanced cancer and to understand how these influence functional behaviours, with the aim of developing clinical recommendations to inform patient care.

Design Participants underwent in-depth, semi-structured, qualitative interviews. Data was analysed using Interpretative Phenomenological Analysis to identify emergent themes.
Setting/Participants: Ten patients with advanced stage cancer experiencing cancer-related pain were recruited from an InnerLondon hospice palliative care service.

Results Meaning of pain in advanced cancer is inextricably linked with loss and bereavement. Meaning of pain is a dynamic, temporal process underpinned by the following themes: (i) nature of cancer-related pain, (ii) multidimensional impact of pain on patients' function and behaviour, (iii) 'Web of Loss': a complex network of losses that perpetuates cycles of further loss and (iv) hope and appreciation of life. The data suggests meaning of pain has an indirect influence on behaviour mediated by the coping strategies patients adopt. Meaning of pain has potential to promote both constructive and maladaptive responses in functional behaviour.

Conclusions Findings further our understanding of the experience of cancer-related pain and inform bio-behavioural approaches to pain management in palliative care. Recommendations include (i) tailoring pain management to dedicate greater attention to the behavioural dimension of cancer-related pain (ii) timely identification and management of preparatory grief in patients with advanced cancer and (iii) promotion of constructive coping strategies to support patients to make sense of their pain and maintain functionality within the limitations of advancing disease.

\section{P162 LOST IN TRANSLATION; PHYSIOTHERAPISTS' ATTITUDES AND BELIEFS TOWARDS PALLIATIVE CANCER PATIENTS IN THE ACUTE HOSPITAL SETTING}

Helen Hutchinson. Princess Alice Hospice, Esher, UK

\subsection{6/bmjspcare-2013-000591.184}

Abstract Aim The study aimed to elucidate any attitudes acute hospital physiotherapists may have towards palliative cancer patients.

Background With advances in medical technology, increasing numbers of patients are 'living with' a terminal cancer diagnosis for longer periods; this coupled with changes in government policy, is resulting in patients being treated closer to home rather than in specialist centres, bringing physiotherapists into increasing contact with this patient group.

Methods Semi-structured interviews (analysed utilising Colaizzi's method of analysis), of the first twelve physiotherapists from an acute hospital in the UK who volunteered for the study and who met the inclusion criteria.

Results Two clusters of themes were identified, namely Attitudes and Issues. There were four sub-divisions of Attitudes; terminology, physiotherapists' perceptions, other professionals' perceived attitudes towards physiotherapists, and patients and their families perceived attitudes towards physiotherapists' role with terminally ill cancer patients.

"I'd say there are a lot of physio's that think it's probably a little bit pointless"

"try and maintain their quality of life ... by their thinking... and hopefully try and meet it."

There were six sub-divisions of issues: communication, education, emotions, boundaries, referring onward and time.

"I think they're [physiotherapists] scared that that's going to be asked of you...will I walk again?"

Conclusions Some physiotherapists understand and apply their skills to improve the quality of life of palliative cancer patients. However it is suggested that the majority of physiotherapists regard these patients as 'dying from' rather than 'living with' a 